\title{
Discovery of novel RyR2 inhibitors as candidates for anti-arrhythmic drugs
}

Mai Tamura ${ }^{1}$, Nagomi Kurebayashi ${ }^{1}$, Takashi Murayama ${ }^{1}$, Shuichi Mori ${ }^{2}$, Mari Ishigami-Yuasa ${ }^{2}$, Hiroyuki Kagechika ${ }^{2}$, Junji Suzuki ${ }^{4}$, Kazunori Kanemaru ${ }^{3,5}$, Masamitsu lino ${ }^{3,5}$, Takashi Sakurai ${ }^{1}$

${ }^{I}$ Department Pharmacology, Faculity of Medicine, Juntendo University, Japan, ${ }^{2}$ IBB, Tokyo Med. Dent. Univ., Tokyo 101-0062, Japan, ${ }^{3}$ The Univ Tokyo, Tokyo 113-0033, Japan, ${ }^{4}$ Univ. California, San Francisco, USA, ${ }^{5}$ Nihon Univ. Sch. Med., Tokyo 173-8610, Japan

Background: The type 2 ryanodine receptor (RyR2) is a $\mathrm{Ca} 2+$ release channel on the endoplasmic reticulum (ER) and plays a central role in the excitation-contraction coupling in the heart. Amino acid mutations in RyR2 are known to cause various types of arrhythmic diseases such as catecholaminergic polymorphic ventricular tachycardia (CPVT). Because spontaneous $\mathrm{Ca} 2+$ release from ER Ca2 + store, which induce triggered activity, is likely to occur in cardiac cells in patients with CPVT, drugs that suppress RyR2 are expected to have antiarrhythmic effects. We have previously indicated that the ER Ca2+ signal in HEK293 cells expressing mutant RyR1 and RyR2 well reflects their Ca2+ releasing activity (Murayama et al, Human Mut., 2016, Uehara et al. J. Gen. Physiol., 2017, Fujii et al. Heart Rhythm, 2017). In this study, we aimed to search for RyR2 inhibitors by ER Ca2+ monitoring using HEK293 expression system and to examine their effects on $\mathrm{Ca} 2+$ signals in cardiac and non-cardiac cells expressing mutant RyR2s.

Methods: HEK293 cells stably expressing wild-type or mutant RyR2 and R-CEPIA1er were generated and the RCEPIA1er signal was measured in a 96-well plate by a FlexStation II spectrophotometer. Effects of RyR2 inhibitor candidates were evaluated by single cell $\mathrm{Ca} 2+$ and/or action potential imaging of HEK293 cells and cultured cardiomyocytes (HL-1 cells, a mouse atrial muscle derived cell line, and human iPS cell-derived cardiomyocytes). Ca2+dependent $\mathrm{Ca} 2+$ release activity of RyR2 was determined by $[3 \mathrm{H}]$ ryanodine binding assay.

Results and discussion: By screening of approximately 1,600 of well-characterized compounds, we identified four compounds that increased the R-CEPIA1er signal over the set reference value (mean value $+4 \mathrm{SD}$ ). All four hit compounds decreased frequency of spontaneous Ca2+ oscillations in HEK293 cells expressing WT and mutant RyR2s and correspondingly reduced the $\mathrm{Ca} 2+$-dependent $[3 \mathrm{H}]$ ryanodine binding. Three out of four compounds did not inhibit generation of action potential in cardiac muscle derived cell lines. These results indicate that the three compounds are promising candidates for novel anti-arrhythmic drugs and that the screening with ER Ca2+ monitoring is a useful method for searching RyR2 inhibitors. 